\section{Adaptive Classification Algorithm for EMC Compliance Testing of Electronic Devices}

\section{P. Singh, D. Deschrijver, D. Pissoort and T. Dhaene}

This letter describes a novel technique that facilitates near-field (NF) scanning for EMC-compliance testing. It performs measurements in a sequential way with the aim of discovering multiple, possibly disjoint regions where the amplitudes of a near-field component belong to certain output ranges. The measured data samples are used to train a classification model where each NF range is represented by a given class (e.g. low/medium/high NF amplitudes). The outcome of the algorithm is a visual map that clearly characterizes and pinpoints the exact location and boundaries of each class. Such maps are useful, for example, to detect hotspots or regions that are prone to electromagnetic compatibility issues. The technique is validated on a measured microstrip bend discontinuity.

Introduction: The detection of electromagnetic compatibility (EMC) issues for electronic devices is becoming important as an increasing amount of components are operating at higher clock frequencies. A popular method to examine the EMC behavior of such electronic (sub)systems is based on electromagnetic near-field scanning [1], as it facilitates the assessment of both the intra-system and inter-system EMC behavior of the device under test. In order to reduce the overall NF scanning time, an automated algorithm was proposed in [2] that builds a high-resolution carthography model of the NF pattern by performing a minimum amount of measurements and fitting the data with a Kriging-based interpolation model. Such models are highly accurate in the sense that they capture all variations in the NF pattern. For EMC pre-compliance testing, however, one is usually interested in identifying only those regions of interest that belong to a certain class (e.g. regions with low/medium/high $\mathrm{NF}$ amplitudes). The early detection of such regions during the designphase yields insight into the EMC behavior of the device and can be exploited to reduce development costs and design effort while increasing the performance. This letter presents a novel classification-based scanning algorithm that sequentially performs batches of measurements with the aim of detecting only the regions of interest and outlining their boundaries. Numerical results confirm that the amount of measurements can be reduced significantly as compared to a full device scan as was proposed in [2].

Goal Statement: The goal of the algorithm is to identify all NF regions of the device that correspond to a given class, while minimizing the number of measurements (data samples) needed. Each data sample, $k$, is conveniently represented as a tuple $\left(\mathbf{x}_{k}, L\left(\mathbf{x}_{k}\right)\right)$ where $\mathbf{x}_{k}$ is a scan point that contains the horizontal, vertical, and height coordinates of the probe and $L\left(\mathbf{x}_{k}\right)$ is the resulting class label. Note that this label is assigned by inspecting the output range of the amplitude of the electric or magnetic field component and by mapping it onto the corresponding class (e.g. low, medium or high).

Adaptive Classification Algorithm: The adaptive algorithm starts from a small initial set of scan points that is chosen according to an optimized latin hypercube design. In each sequential step, optimal coordinates for an additional batch of NF scan points are calculated and the corresponding measurements are performed. This process is repeated iteratively until all the regions of interest are well characterized. The resulting data samples are used to train a classification model (e.g. Least-Squares Support Vector Machine (LS-SVM), Artificial Neural Network (ANN),...) that predicts the class label at arbitrary coordinates in the scanning plane. The predictions of the model can be used to calculate a visual map that accurately pinpoints the location and the boundaries of each class region.

Sequential Sampling Scheme: When choosing scan points, it is important that exploration and exploitation criteria are combined in a balanced way.

- Exploration makes sure that the scanning plane is sampled as evenly as possible, to ensure that no important regions are missed. Given a set of $K$ scan points, a Voronoi tessellation is computed that divides the plane into cells $C_{k}$. Cells with a larger relative volume correspond to sparse areas and are assigned a higher score $V\left(\mathbf{x}_{k}\right)$ (see Sect. III-A in [2]).

$$
V\left(\mathbf{x}_{k}\right)=\frac{\operatorname{Vol}\left(C_{k}\right)}{\operatorname{Vol}\left(C_{1}\right)+\ldots+\operatorname{Vol}\left(C_{K}\right)}
$$

\section{ELECTRONICS LETTERS 3rd August 2013 Vol. $00 \quad$ No. 00}

- Exploitation makes sure that the boundaries between different classes are sampled more densely, because these are the areas where the uncertainty of the classification model is the largest. For each scan point $\mathbf{x}_{k}$, a neighbourhood $\left\{\mathbf{x}_{k}^{n}\right\}_{n=1}^{N}$ of $N$ scan points is calculated (see Sect. 4.2 in [3]). These neighbours should satisfy the properties of cohesion and adhesion. Cohesion implies that neighbours are located as close to the scan point as possible, while at the same time adhesion implies that the neighbours must be as far apart from each other as possible. These properties ensure that all directions around the scan point are well covered. Once the neighbourhood is constructed, the class labels $L\left(\mathbf{x}_{k}^{n}\right)$ of all neighbours are compared. A higher score $W\left(\mathbf{x}_{k}\right)$ is assigned to Voronoi cells where class labels of the neighbours have a mismatch, because this corresponds to the areas where boundaries are located.

$$
W\left(\mathbf{x}_{k}\right)=\left\{\begin{array}{l}
1 \Longleftrightarrow \forall 1 \leq i, j \leq N: L\left(\mathbf{x}_{k}^{i}\right)=L\left(\mathbf{x}_{k}^{j}\right) \\
0 \Longleftrightarrow \exists 1 \leq i, j \leq N: L\left(\mathbf{x}_{k}^{i}\right) \neq L\left(\mathbf{x}_{k}^{j}\right)
\end{array}\right.
$$

Once these metrics are computed for each Voronoi cell, all the cells are ranked according to a global metric $G(\mathbf{x})$ that combines both the criteria

$$
G(\mathbf{x})=V(\mathbf{x})+W(\mathbf{x})
$$

Optimal scan coordinates for the next batch of scan points are then chosen inside the highest ranked cells, and in such a way that the distance from the neighbours is maximized. The iterative process of measuring additional scan points is repeated until all the class regions are well characterized.

Experiment: The algorithm was applied to scan the NF pattern of a double bended microstrip line (see Fig. 1) that was measured using a NF scanning system. The PCB comprised of a $50 \Omega$ microstrip on a $15 \mathrm{~cm} \times 9 \mathrm{~cm}$ FR4 substrate with a thickness of $1.5 \mathrm{~mm}$. To create sufficient radiation, some basic EMC rules were violated on the test PCB (e.g., routing the microstrip over a slot in the ground plane). The microstrip was excited at one end with a generator set at an available power of $0 \mathrm{dBm}$ and terminated in $50 \Omega$ at the other end. The amplitude of a field component, e.g. $\left|H_{y}\right|$, was measured with a NF scanner that was built from a computer numerical control milling machine. To do this, the miller and its suspension were removed and replaced by a head to which a near-field probe is attached. The head can be moved automatically in three dimensions above the device under test to perform measurements. The probe was a magnetic NF probe from Langer EMV-Technik (RF-U 2.5-2) that was specified for the frequency range of [30 MHz - $3 \mathrm{GHz}$. It was connected to a Rohde \& Schwarz EMI receiver and all the measurements were performed at a fixed height of $2 \mathrm{~mm}$ above the printed circuit board (PCB) under test at a frequency of $200 \mathrm{MHz}$.

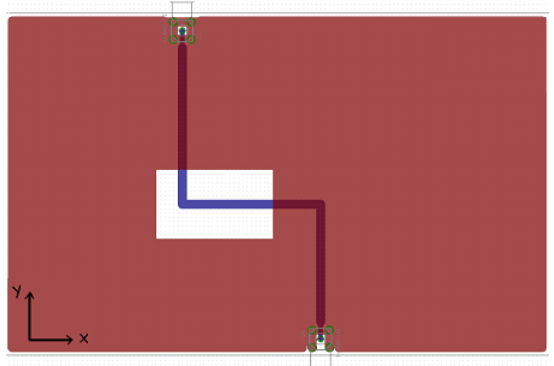

Fig. 1. Layout of the bent microstrip

The algorithm is used to detect 3 regions in the scanning plane where the amplitude of the NF component $\left|H_{y}\right|$ corresponds to a different level:

\begin{tabular}{|c|c|c|}
\hline Class label & NF Range $(\mathrm{dB} \mu \mathrm{V})$ & Colour \\
\hline \hline Low & {$[0-30[$} & blue \\
\hline Medium & {$[30-40[$} & green \\
\hline High & {$[40-\infty[$} & red \\
\hline
\end{tabular}

It starts from an initial set of 80 measurements that are chosen according to an optimized Latin Hypercube. The sampling algorithm iteratively computes the optimal coordinates of a batch of 20 additional scan points and the measurements are performed. This process is repeated until a total amount of 700 samples are available and LS-SVM classification model is calculated whose output is visualized as a map in Fig. 2. It is seen that the algorithm focuses the location of selected scan points (black dots) near the 
class boundaries where the classifier is rather prone to uncertainty, while at the same time covering the overall region in a space-filling way [4].

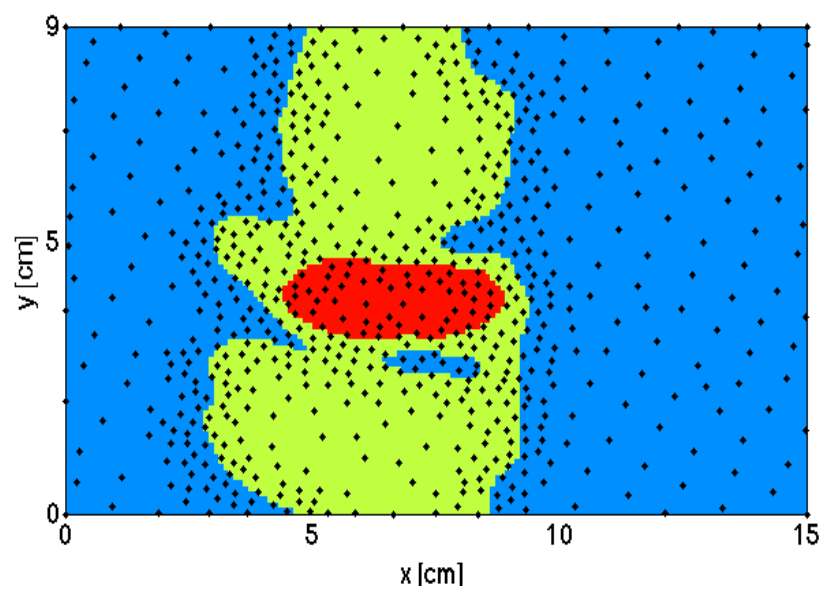

Fig. 2. Map of classification model with 700 scan points marked as black dots

The same experiment was repeated for varying number of scan points, and the resulting models are validated on a separate test set of 500 measurements that are uniformly distributed in the scanning plane. Table 1 shows that the resulting models perform very well in terms of precision, recall and accuracy, even with a limited number of scan points (150 or above). This is a significant reduction when compared to a full device scan that would require at least 350 measurements (see Fig. 22 in [2] for details).

\begin{tabular}{|l|l|l|l|}
\hline \# Measurements & Precision & Recall & Accuracy (\%) \\
\hline 100 & 0.5702 & 0.6191 & 89 \\
\hline 150 & 0.9523 & 0.9023 & 95 \\
\hline 200 & 0.9676 & 0.9245 & 96.6 \\
\hline 250 & 0.9679 & 0.9154 & 96.8 \\
\hline 300 & 0.9622 & 0.9460 & 96 \\
\hline 700 & 0.9865 & 0.9865 & 98.4 \\
\hline
\end{tabular}

Table 1: Validation of the classification models.

Conclusion: A novel algorithm is proposed for EMC-compliance testing of electronic devices by means of NF scanning. It combines adaptive classification and sequential sampling algorithms to iteratively identify the regions of interest where the amplitude of the NF pattern has prespecified characteristics. Numerical results show that it can significantly reduce the number of measurements needed when compared to a full device scan.

Acknowledgment: This research has been funded by the IWT (Flanders, Belgium) through the Technology Transfer project NEATH, by the Interuniversity Attraction Poles Programme BESTCOM initiated by the Belgian Science Policy Office, and the Research Foundation Flanders (FWO-Vlaanderen). Dirk Deschrijver is a post-doctoral researcher of FWO-Vlaanderen.

P. Singh, D. Deschrijver and T. Dhaene (Ghent University - iMinds, 9000 Ghent, Belgium); D. Pissoort (University College Katholieke Hogeschool Brugge-Oostende, B-8400 Ostend, Belgium)

E-mail: (prashant.singh, dirk.deschrijver, tom.dhaene)@intec.ugent.be, davy.pissoort@khbo.be

\section{References}

1 Baudry, D.; Archambal, C.; Louis, A.; Mazari, B. and Eudeline, P.: 'Applications of the near-field techniques in EMC investigations', IEEE Trans. Electromagn. Compat., vol. 49, 2007, 3, pp. 485-493

2 Deschrijver, D.; Vanhee, F.; Pissoort, D. and Dhaene, T.: 'Automated NearField Scanning Algorithm for the EMC Analysis of Electronic Devices' IEEE Trans. Electromagn. Compat., vol. 54, 2012, 3, pp. 502-510

3 Crombecq, K.; Gorissen, D.; Deschrijver, D. and Dhaene, T.: 'A Novel Hybrid Sequential Design Strategy for Global Surrogate Modeling of Computer Experiments' SIAM Journal on Scientific Computing, vol. 33, 2011, 4, pp. 1948-1974
4 Gorissen, D.; Couckuyt, I.; Demeester, P.; Dhaene, T. and Crombecq, K.: 'A Surrogate Modeling and Adaptive Sampling Toolbox for Computer Based Design' The Journal of Machine Learning Research, vol. 11, 2010, pp. 2051-2055. 